\title{
PHOTOSTRESS RECOVERY TIME AMONG NIGERIANS
}

\author{
BY \\ *OMOKHUA, P. O. AND GEORGE, O. G. \\ DEPARTMENT OF OPTOMETRY, FACULTY OF LIFE SCIENCES \\ UNIVERSITY OF BENIN, EDO STATE, NIGERIA \\ Email:paulohing@yahoo.com \\ *Corresponding author
}

\section{ABSTRACT}

Visual impairment may result from a deficiency of the retinal pigment epithelium or macula. Macula photostress test (MPST) is a quantitative estimation of the functional capability and performance reserve of the photoreceptive elements of the macula/retina. The purpose of this study was to determine the Photostress Recovery Time (PSRT) of different age groups in a sample of healthy subjects. Two hundred subjects with an age range of 11 years to 70years (mean age of 33.30 \pm 12.74 years) were used for this study. All the subjects had clear ocular media, normal appearance of the fundus, and visual acuity of $6 / 6$ or better in the tested eye. Prior to experimentation each subject was adapted to the ambient room lighting for about 5mins. The upperlid of the eye to be tested was held firmly while a penlight was shone into the eye for about 10 seconds, PRST was assessed immediately after 10 seconds by asking the subjects to read at least three letters on the V.A line immediately above the subject's best acuity line. The testing order of subjects in different age groups was randomized. Results indicated that the PSRT distribution in the sample population was found to be positively skewed with a mean of PSRT ranging from 10.34seconds to 46.98 seconds for an age range of 11 years to 70 years. Clinically males had slightly higher PSRT when compared to females. There was a positive relationship between age and PSRT which was highly significant $(\mathrm{r}=0.96)$ with a linear regression equation of PSRT $=0.785+0.60 x$ age. The mean increase in PSRT across consecutive age groups investigated was $27.46 \%$. Males demonstrated a much sharper and larger increase in PSRT across consecutive age groups when compared to the females at all ages investigated except in the presbyopic age group. This study attempts to establish age related PSRT scores among healthy Nigerians in order to provide a quick reference in comparing normal physiological PSRT values and PSRT values arising from impending retinal/macular lesions

KEYWORDS: Macular photostress test, Photostress recovery time, Retinal photoreceptors, Retinal pigment epithelium

\section{Received 25/03/2010}

Accepted 24/05/2010

\section{INTRODUCTION}

Macular photostress test (MPST) is a simple test that can be used in differentiating the cause of reduced visual acuity in an eye arising from a lesion either in the optic nerve or in the retina/macula. Photostress is a psychophysical process which should persist after light exposure, this is because photostress induces an afterimage in the form of a scotoma which subsequently recedes after which normal vision is restored ${ }^{1}$. Optic nerve dysfunction does not affect the PSRT but retinal diseases do. Photostress Recovery Time (PSRT) is the time recorded as the period between when the light is removed and the subject can again begin to read the optotypes on the visual acuity chart just above the initial acuity.

The recovery from photostress is dependent upon the rate of photopigment resynthesis as well as the functional relationship between the photoreceptors and the retinal pigment epithelium (RPE) ${ }^{1}$. Glaser et $\mathrm{al}^{2}$ used a penlight to dazzle the eye to determine the PSRT of his subjects; his results corresponded with that of Severine et $\mathrm{al}^{3}$, who used an elaborate high intensity light flash system. PSRT has been found to increase slightly with age ${ }^{2,4}$. The implication of an age-dependent reduction in PSRT is that there is a nonpathologic deterioration of macular function with age.

There appears to be a wide variation in the average photostress recovery time among different studies. Chilaris reported $10-50$ secs $^{5}$, Glaser $27 \pm 11$ secs $^{2}$ and Sherman $41.97 \pm 17.34$ secs $^{6}$. The lack of standardization with respect to the intensity and duration of the bleaching light, the method used to measure visual acuity, the chosen end point of the test and the population studied could 
undoubtedly account for this wide variation in PSRTs.

Margrain and Thompson ${ }^{7}$, carried out an investigation on the source of variability in clinical photostress test, they evaluated the effect on photostress technique, age, ametropia, pupil size, and acuity on PSRT in normal subjects when they used a reference technique designed to bleach a consistent amount of photopigment. Analysis of data obtained with the reference and best clinical technique showed that age was the only factor that had a significant effect on PSRT. However patients with different pathologies ranging from maculopathies, glaucoma, retinitis pigmentosa, multiple scleroses, optic neuritis and optic atrophy, showed an abnormal(longer) response after photostress with respect to age-matched controls ${ }^{8}$.

Since standard clinical tests of visual function are directed at measuring thresholds of various visual functions and not their performance reserve, a thorough assessment of visual function should, therefore, include dynamic testing procedures such as the MPST especially when the results of standard tests do not adequately explain a patient's visual symptoms. Comparison of an individual's PSRT rates with age-related norms should provide an index of the "physiological age" of macular or cortical function. Significant discrepancies between a patient's "chronologic" age and "physiologic age" should be viewed with suspicion as they may indicate an incipient ocular disease. The purpose of this study was to establish age related normative values of PSRT among healthy Nigerian population of different age groups and investigate if any gender differences exist in the distribution of such values among the study population.

\section{RESEARCH METHODOLOGY}

Two hundred subjects (110 females and 90 males), with age ranging from 11 to 70 (mean age of $33.30 \pm 12.74$ ) years, participated in this study. All subjects were of pure stock Nigerian parentage and had clear ocular media, normal appearance of the fundus, and visual acuities of $6 / 6$ or better either aided or unaided. Subjects were literate enough to read the Snellen's chart fluently in the test eye. Prior to experimentation each subject was adapted to the ambient room lighting for about 5 mins.

The MPST was performed according to a modified technique of Glaser et al. ${ }^{3}$ First, the visual acuity was measured for the eye to be tested using illuminated Snellen optotypes and then the eye with a normally reactive pupil was dazzled with a penlight held approximately $2.0 \mathrm{~cm}$ from the cornea for 10 seconds. In order to minimize variations in penlight intensity and their effect on PSRTs, the penlights used over the course of this study produced an illuminance of 2,340 lumens $/ \mathrm{m}^{2}$. The batteries of the penlight were charged after being used for 15 subjects (for 150 seconds). An 'Energizer' penlight with halogen bulb was used. It was powered with two Duracell alkaline size AA $1.5 \mathrm{~V}$ batteries. A photocell was used to test the voltage of the batteries to ensure standardization. The testing order of subjects in different age groups was randomized.

During the photostress procedure each subject looked directly into the centre of the penlight. If the subject wore glasses, they were lowered on the nose during the photostress period and quickly repositioned into the normal spectacle plane as the subject attempted to read the letters in the visual acuity chart, this was to prevent reflection. The dominant eye was detected and tested in all subjects, the upper eyelid of the eye was held up by the examiner against the superior orbital rim, while the penlight was shone with the other hand (this was done to prevent blinking or shutting of the lid by the subject during dazzling). An electronic stopwatch with a tone so programmed was used and the commencement of timing alerted the examiner to shine the light into the subject's eye. The timing was done by an assistant who had been instructed by the examiner in the procedure.

Immediately after removing the penlight, the subject was asked to begin reading the test letters on the line just above the best acuity line. The time that elapsed between the end of the photostress period and the time when the subject could correctly identify two or more letters above his prestress best acuity line was recorded as the PSRT.

\section{QUALITYASSURANCE}

Reproducibility of PSRT value was tested in 15 subjects. The initial mean PSRT was $22.9 \pm 15.75$ seconds while the repeats mean PSRT was 22.21 \pm 5.70 . Paired t-test $(\mathrm{t}=1.665, \mathrm{P}>0.05)$ indicated no significant difference between both procedures.

\section{RESULTS}

The mean age and standard deviation of the subjects tested were $33.30 \pm 12.74$ years. The observed distribution of PSRT of the sample population ranged from 8 seconds to 47 seconds. At 
95\% confidence interval, the mean PSRT ranged from 10.34 seconds to 46.98 seconds for an age range of 11 years to 70 years. Fig 1 below shows the age distribution of subjects according to gender in the sample population. Chi-square goodness of fit for uniform distribution indicate no significant difference $\left(X^{2}=2.00\right)$ in the number of males and females used in the sample population.

As shown in fig. 2 the PSRT was found to be associated incrementally with increasing age, suggesting a nonpathologic deterioration of macular function with age. Males tended to have slightly higher PSRT when compared to females across most age groups investigated as shown in fig. 3. Mean PSRT across all age groups investigated for males and females respectively were $25.77 \pm 12.49$ and $25.04 \pm 11.83$, but unpaired t-test indicated no significant difference in PSRT scores between gender $(\mathrm{t}=1.03, \mathrm{P}<0.05)$. Fig. 4 shows the distribution of PSRT in the sample population to be positively skewed with a skewness of +0.751 (more values towards the right), the PSRT score of 12 seconds accounted for the highest frequency in the sample population. The scatter diagram in fig. 5 indicates that a positive relationship existed between age and PSRT which was highly significant $(r=0.96)$ with a linear regression equation of PSRT $=0.785+$ 0.60xage. Fig 2 depicts the mean PSRT at 95\% confidence interval and the corresponding Standard Error of Mean (SEM) across the different age groups investigated, the mean of the age group of 11-20 years accounted for the closest population mean, while the age group of 51-60 years demonstrated a mean farthest from the population mean.

Fig. 6 shows the percentage increase in PSRT scores between consecutive age groups investigated in the study. The mean consecutive increase of PSRT across all age groups investigated was $27.46 \%$. The PSRT between the consecutive age groups of 51-60 years and 61- 70 years showed the greatest percentage change in PSRT, while the PSRT between the age groups of 11-20 years to 21-30 years showed the least percentage reduction in PSRT. There was no significant difference among males and females in the change in PSRT scores between consecutive age groups in the sample investigated. In Fig. 7 the trend in the consecutive change in PSRT with age showed that between ages of 11years to 40 years the males demonstrated a much sharper and larger increase in PSRT when compared to the females who demonstrated a lesser but gradual increase in
PSRT. Between ages 40years to 50years there was a huge drop of PSRT in males and they actually presented with a clinically lesser PSRT when compared to females. Between ages 50years to 60years the males then demonstrated a steep increase in PSRT to become higher than the females. All through the ages investigated the females demonstrated a gradual increase in PSRT up till 60 years and above which presented with the sharpest increase. At age 60 years and above the males also presented with the sharpest increase in PSRT, thus coinciding with the pattern presented by their female counterparts, though the PSRT of males were still slightly higher.

\section{DISCUSSION}

Though a wide range of normal results have been reported in the literature (and this perhaps is the reason that MPST has been of little use by clinicians) it does not dim the significance of the Macular Photostress Test in distinguishing between retinal and post-retinal diseases. In this study a simple, reproducible and readily available method of determination of PSRT was used. This study attempted to establish PSRT baseline scores for Nigerians in different age groups in order to provide PSRT scores that would give an indication of the normal "physiological aging" of macular/retinal function. This study also provided the pattern of PSRT values that existed among males and females in the same age groups.

From the age groups (11 to 70 years) investigated a PSRT range of approximately 10 seconds to 47 seconds represented the lower and upper boundaries for a normal functional retina/macula among Nigerians. PSRT beyond the upper limit of the confidence interval for each age group (11.57secs for 11-20years, 17.10secs for 21$30 y e a r s, 23.28 \mathrm{secs}$ for $31-40 y e a r s, 27.87 \mathrm{secs}$ for 41.50years, $36.61 \mathrm{secs}$ for 51-60years and 46.98secs for 61-70years) investigated would suggest an impending pathologic problem among Nigerians. The increase in PSRT score with increasing age recorded in the study would indicate that as one ages, it takes more time for the photoreceptive elements to return to their prebleach acuity level. This further translates to a gradual nonpathologic deterioration of the macula. Though not statistically significant but of clinical importance, males had a longer PSRT scores when compared to females for the age groups investigated as shown in fig 1 .

Since increase in PSRT is a normal physiological decline in visual function, we suggest that anatomical andlor neurological 
changes that might account for this decline may vary slightly in males when compared to females. The distribution of the PSRT scores though a reflection of the age distribution of the sample population, is shown to be positively skewed towards increasing age indicating that in the general population, fewer individuals have higher PSRT scores which has been shown as a function of ageing (fig 5.) PSRT scores were highly correlated with age $(r=0.96)$ and predictability of PSRT scores from the age of the subject was given by the coefficient of regression; $y($ PSRT $)=$ $0.785+0.60 \times$ (age).

This study thus brings to the fore PSRT as most prominent of the three known ocular parameters (lacrimation, accommodation and fluoresence of the cornea and lens) for the non-invasive assessment of an individual's age ${ }^{9}$. Fig. 2 also shows the standard error of mean and the confidence interval (95\%) for PRST scores. From the sample population there was less variability in PSRT scores when compared to the general population in the younger age groups, this was evident from the small standard error of mean recorded, thus indicating a precision of mean whose results could be extrapolated to the general population. Varied age related effects can be suggested to account for the large standard error of mean and a consequent higher variability as noticed in the distribution of PSRT for much older age groups (51-60 years and 61-70 years) in the sample population as when compared to the general population. The mean increase in PSRT across consecutive age groups investigated was $27.46 \%$, implying there was a nonpathologic deterioration of the retinal $\backslash$ macular photoreceptive elements of about $27.46 \%$ across two decades of life in a healthy retina.

Fig. 6 shows each percentage change across consecutive age groups, the greatest changes were

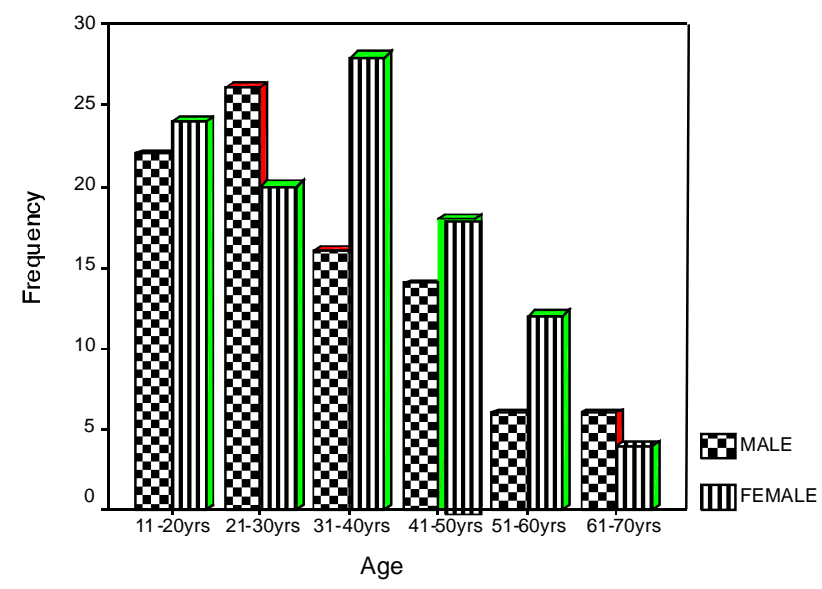

Fig.1: Age distribution by gender in the sample population noticed in the age groups of 51-60years to 6170 years $(40.89 \%)$ and the age groups of $41-50$ years to $51-60$ years $(30.52 \%)$ while the least change $(19.57 \%)$ was recorded in the $11-20$ years to 21-30 years age group. Since the other ocular media apart from the crystalline lens and macular pigment vary very little in light transmittance with age, the age related changes seen in PSRT would be a function of both or either the crystalline lens and/or macular pigment. Sample et $\mathrm{al}^{10}$ had shown that a similar pattern of change exists when lens density index was plotted as a function of age. These changes have been suggested to be attributed to absorption of light as a result of yellowing of the lens.

In this study as depicted in fig. 7 a change in pattern between consecutive age groups change in PSRT was quite evident in the age groups that coincide with presbyopia, this was quite evident in the presbyopic male group, re-enforcing the fact that the crystalline lens might play a role in PSRT scores. As to the clinical variability in the change in consecutive PSRT between male and females in this presbyopic age group, gender differences in the onset of presbyopia in the Negroid population may account for these changes noticed in the study $^{11}$

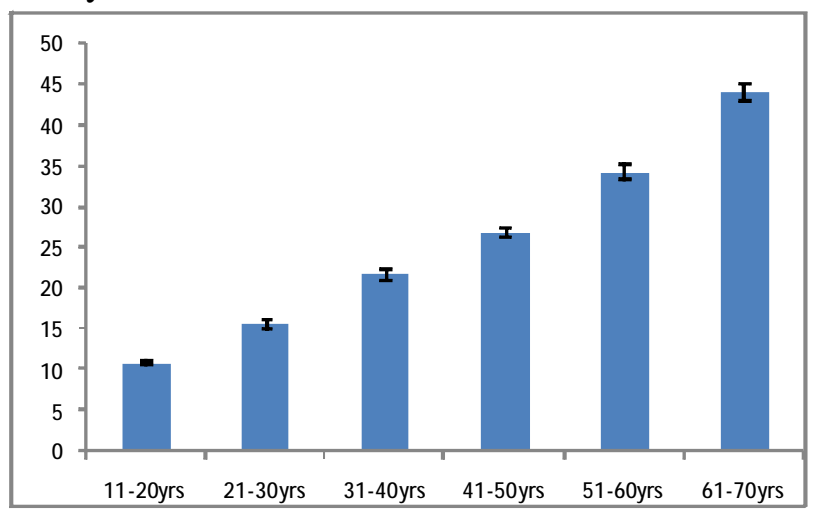

Fig. 2: Age distribution of mean PSRT (with standard Error of mean bars in the sample population

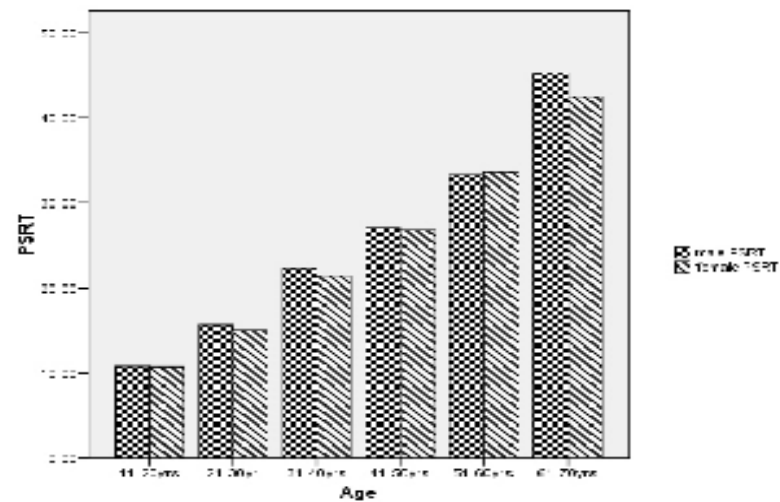

Fig. 3: Distribution of PSRT scores by gender in the sample population 


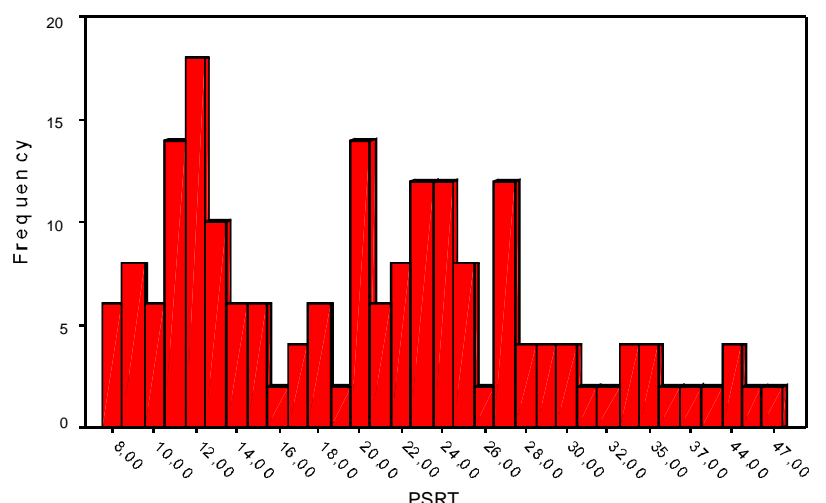

Fig. 4: Distribution of PSRT scores in the sample population

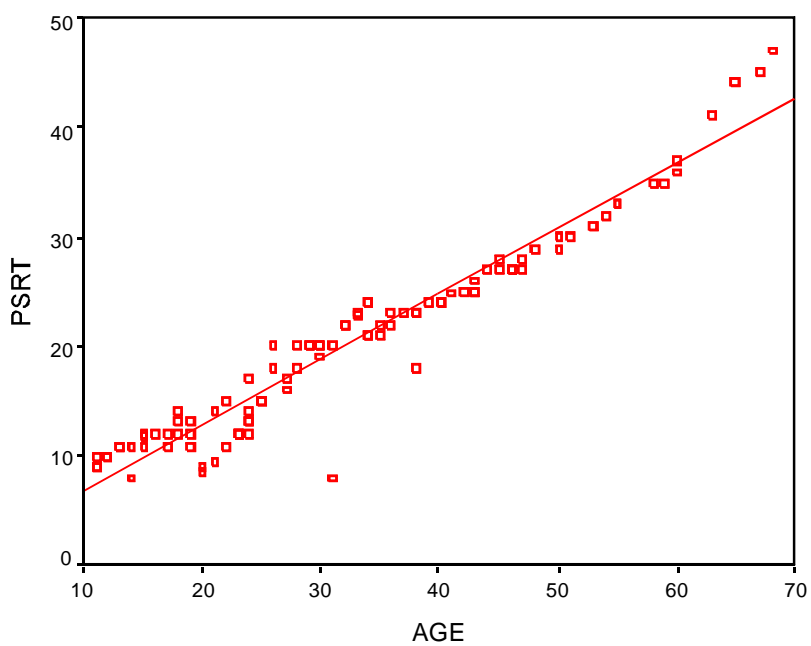

Fig. 5: Relationship between PSRT and Age in the sample population

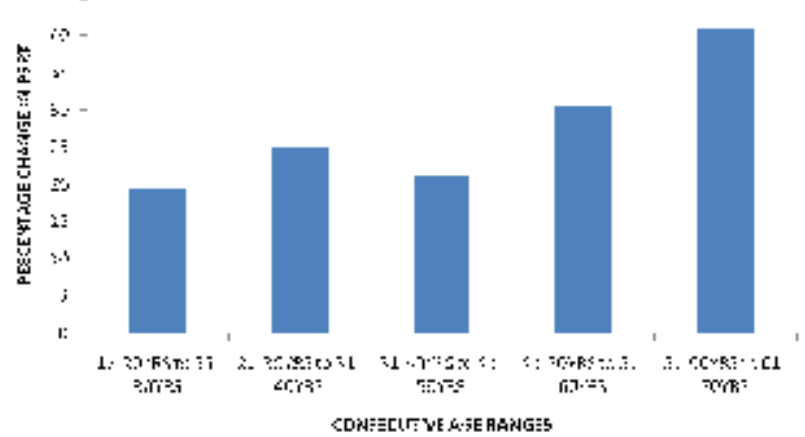

Fig. 6: Percentage reduction of PSRT between age groups in the sample population

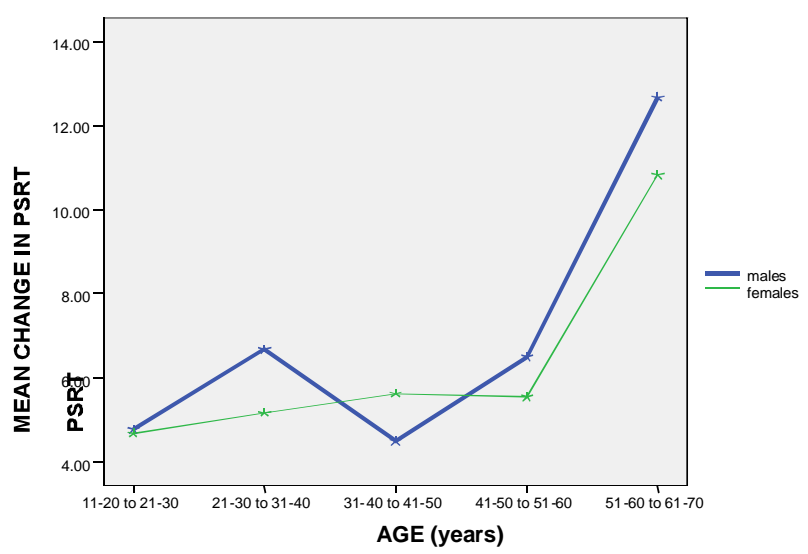

Fig. 7: Change in consecutive PSRT scores between male and females across different age groups

\section{REFERENCES}

1. Hackenlively, J. R., Pearlman, J. T., Shaver, L., Brickman, M. and Henkind, P. (1998): Macular Recovery from dazzle (photostress) in normal women. Doc. Ophthalmol. Proc. Ser, 15:313-6.

2. Glaser, J. S., Sarino, K. D., McDonald, S. A. and Knighton, R.

W. (1979): The photostress recovery test in the clinical assessment of visual function. Am. J. Ophthalmol, 83: 255-60.

3. Severine, S. I., Tour, R. I. and Kershaw, R. H. (1967): Macular

function and the Photostress Test 2. Arch. Ophthalmol, 77:163-7.

4. Dhalla, M. S. and Fantin, A. (2005): Macular photostress

testing. Br. J. Ophthalmol, 25(2): 189-92.

5. Chilaris, G. A. (1962): Recovery time after macular illumination

as a diagnostic and prognostic tool. Am. J. Ophthalmol, 53:311-4.

6. Sherman, M. D. and Jenkins, P. (1988): Photostress recovery in chronic simple Glaucoma. Br. J. Ophthalmol, 72: 641-5.

7. Magrain, T. H. and Thomson D. (2002): Sources of variability in

clinical photostress test. J. Ophthalmol. Physiol. Opt, 22: 61-7.

8. Dhalla, M. S., Fantin, A., Blinder, K. J. and Bakal J.A. (2007):

The Macular Automated Photostress Test. Am. J. Ophthalmol, 143(4): 596-600.

9. Weale, R. (2001): Vision and anatomical changes with aging

eye. Lancet, 358:1644-6.

10. Sample, A. P., Esterson, F. D., Weinreb, R. N. and Boynton, R.

M. (1998): The Aging Lens: In Vivo Assessment of Light Absorption in 84 Human Eyes. Invest. Ophthalmol. Vis. Sci, 29(8): 1306-11.

11. Morny F. K. (1995): Correlation between presbyopia, age, and

number of births of mothers in the Kumasi area of Ghana. Ophthalmic Physiol. Opt, 15: 4636. 